\title{
Perspectivas de la inteligencia emocional y desarrollo del aprendizaje cognitivo.
}

\author{
Balance of payments in Ecuador and its comparison with the balance of \\ payments of Venezuela and Chile of the year 2017
}

Mgs. Nelly Noemí Andrade Albán. ${ }^{1}$, Dr. Mcs. Jorge Rodrigo Andrade Albán. ${ }^{2}$, Md.Esp. Noemí Rocío Andrade Albán. ${ }^{3}$, Dr, PhD. Víctor Peñafiel Gaibor. ${ }^{4} \&$ Mcs.Angel Leonardo Andrade. ${ }^{5}$

Recibido: 01-09-2017 / Revisado 01-11-2017 Aceptado: 11-12-2017/ Publicado: 01-01-2018

\begin{abstract}
.
DOI: https://doi.org/10.33262/cienciadigital.v2i1.24

The research aims to determine the importance of emotional intelligence to strengthen cognitive learning, improving self-esteem, positive expression, supporting emotional development, critical thinking, reducing interpersonal conflicts; being of transcendental in the process of creativity, reasoning, reflection to achieve personal development, increasing through the study the understanding of the competencies to reach the person fullness, integrating the capacities, potentialities being able to solve the environmental problems with autonomy and independence. In the research modality, the qualitative approach was applied in the methodology, when gathering information and favoring the information of the theoretical framework; the quantitative approach by which the use of statistical analysis verifies the acceptance or rejection of the hypotheses applying the formulation of the chi-square; In addition, the study was field-based when establishing a direct relationship between the study variables, determining the causes and consequences of the bibliography when documents were used as sustenance for the writing of the research work. In the levels and types stand out, the explanatory level, descriptive, explanatory and by association of variables; We worked with a population of 49 people studied 43 students and 5 teachers having a

\footnotetext{
${ }^{1}$ Distrito Educativo Intercultural Bilingue, Ambato, Ecuador , nellycym@ hotmail.com

${ }^{2}$ Universidad Técnica de Ambato, Facultad de Ciencias Humanas y de la Educación, jr.andrade@uta.edu.ec

${ }^{3}$ Universidad Técnica de Ambato, Facultad de Ciencias de la Salud, nr.andrade@uta.edu.ec

${ }^{4}$ Universidad Técnica de Ambato, Facultad de Ciencias de la Salud, vi.penafiel@uta.edu.ec

${ }^{5}$ Universidad Técnica de Ambato, Facultad de Ciencias de la Salud, aandrade@ pronaca.com
} 
sample equivalent to a percentage of $100 \%$; the operationalization of the variables was carried out, applying as a technique to the survey directed to students with their instrument the structured questionnaire composed of 10 students; the interview was also used, orienting the interview guide to gather information from the teachers; According to the results obtained, it can be deduced that Emotional Intelligence influences Cognitive Learning.

Keywords: Emotional Intelligence, Multiple Intelligences, Emotional Competences, Cognitive Learning, Theories Of Learning.

\section{Resumen.}

La investigación tiene como finalidad determinar la importancia de la Inteligencia emocional para fortalecer el aprendizaje cognitivo, mejorando el autoestima, la expresión positiva, apoyando en el desarrollo emocional, el pensamiento crítico, disminuyendo los conflictos interpersonales; siendo de trascendental en el proceso de la creatividad, el razonamiento, la reflexión para alcanzar el desarrollo personal, incrementando a través del estudio la comprensión las competencias para alcanzar la plenitud persona, integrando las capacidades, potencialidades siendo capaz de resolver los problemas del entorno con autonomía e independencia. En la modalidad de investigación se aplicó en la metodología el enfoque cualitativo, al recabarse información y favoreció en la información del marco teórico; el enfoque cuantitativo por que mediante la utilización del análisis estadístico se verifica la aceptación o rechazo de las hipótesis aplicándose la formulación del chi cuadrado; además el estudio fue de campo al establecer una relación directa entre las variables de estudio, determinándose las causas y consecuencias fue bibliográfica al emplearse documentos como sustento para la redacción del trabajo de investigación. En las niveles y tipos sobresalen, el nivel explicativo, descriptivo, explicativo y por asociación de variables; se trabajó con una población de 49 personas objeto de estudio 43 estudiantes y 5 docentes teniendo una muestra equivalente a un porcentaje de $100 \%$; se realizó la operacionalización de las variables aplicándose como técnica a la encuesta dirigida a estudiantes con su instrumento el cuestionario estructurado integrado por 10 estudiantes; también se utilizó la entrevista, orientándose la guía de entrevistas a recabar información a los docentes; según los resultados obtenidos, se deduce que la Inteligencia emocional influye en el Aprendizaje cognitivo.

Palabras Claves: inteligencia emocional, inteligencias múltiples, competencias emocionales, aprendizaje cognitivo, teorías del aprendizaje. 


\section{Introducción.}

Dentro del contexto escolar es importante conocer el desarrollo emocional del educando para que puedo obtener una mejor comprensión y como docente saber llegar a los estudiantes para que les puedan contar sus problemas y juntos buscar una solución.

La inteligencia emocional ha suscitado un gran interés en el ámbito educativo como una vía para mejorar el desarrollo socioemocional del ser humano; (Extremera Pacheco \& Fernández-Berrocal, 2004), la importancia de desarrollar habilidades emocionales en el aula, es una tarea aún pendiente en la mayoría de los centros educativos (Brackett, Rivers, Shiffman, Lerner, \& Salovey, 2005) de esta manera se mantendrá un ambiente favorable dentro del aula para que exista interacción y participación por parte de los educandos.

La Inteligencia emocional es primordial dentro del contexto educativo ya que fomentara en el educando las habilidades que contribuirán de forma positiva al bienestar personal y social del alumno, a través de investigaciones se ha determinado que las personas emocionalmente inteligente no sólo son más hábiles a la hora de percibir, comprender y manejar sus propias emociones, sino también son más capaces de extrapolar estas habilidades a las emociones de los demás.

La Inteligencia Emocional es el ajuste para lograr el bienestar personal, (Fernández \& Ruiz, 2008) que favorece en el éxito en la vida y a la vez mejora las relaciones interpersonales dentro del contexto escolar. (Extremera Pacheco \& Fernández-Berrocal, 2004), enfatiza la participación de las emociones en la solución; (Bello, Rionda, \& Rodríguez, 2010) dice que en el ámbito educativo es importante que el docente realice programas de desarrollo de las capacidades emocionales y sociales

El aprendizaje es caracterizado como herramienta importante para el progreso económico, social; (Fernández \& Ruiz, 2008)analiza su valor tanto presente como futuro para las políticas educativas en el sistema al dar inicio en el infante al desarrollo de sus habilidades de inteligencia emocional; mientas (López V. , 2016) expone que la inteligencia aporta para el dominio de emociones propias y las relaciones interpersonales, por lo cual (Ramos E. , 2017) hacen posible la solución de problemas, y el alcance de metas.

Al estimar que el docente es el encargado del desarrollo de la Inteligencia de sus estudiantes, a través de la motivación y el aprendizaje en un ambiente libre y autónomo, para fomentar conductas, hábitos, costumbres de estudio, y alcanzar el desarrollo integral, la investigación propone como objetivos: Determinar la influencia de la Inteligencia Emocional en el aprendizaje Cognitivo, identificar el nivel de inteligencia emocional de los estudiantes de primer año de Bachillerato General Unificado, establecer las causas que 
influyen en el aprendizaje cognitivo y proponer un informe académico o artículo que detalle la relación entre las variables de estudio.

Dentro de la educación la inteligencia emocional es un requisito principal para la vida al aportar para el avance en las capacidades emocionales hasta mejorar las relaciones interpersonales para alcanzar el éxito académico; (Molina de Colmenares, 2002) además los educadores al ser orientadores en la adquisición de saberes tienen como responsabilidad fomentar valores como el respeto a la opinión de los demás para la socialización de conocimientos y experiencias

La inteligencia es sinónimo de memoria, voluntad, sentimiento, entendimiento e intelecto; (Molina de Colmenares, 2002) debido al progreso en la memoria según la evolución en la edad, se constituye en una facultad humana básica para la capacidad para el aprendizaje y con el desenvolvimiento individual y social; (Caballo, 2002), en la adolescencia el educando manifiestan actitudes como agresión física, agresión verbal, hostilidad e ira muestran niveles significativamente más bajos de inteligencia emocional.

Los controles conductual y emocional que componen el constructo estabilidad emocional, están directamente relacionados con la capacidad de sobreponerse a situaciones negativas, (Moreno, 2017), las personas estables emocionalmente demuestran control de impulsos, aceptan mejor las críticas de los demás y la incertidumbre; (Bermúdez, Álvarez, \& Sánchez, 2003), abordan una actuación eficaz, afrontan situaciones, aprenden al superar los fracasos.

La inteligencia es elemental ya que incluye aspectos afectivos que determinan el reconocimiento de sí mismo, la expresión de los sentimientos, el equilibrio afectivo; elementos que pueden englobarse bajo el concepto de competencias emocionales y el componente social se refiere a las relaciones con las personas en las instituciones, en las que cada uno vive y se desenvuelve (Torregrosa, García, Martínez, \& Delgado, 2014)

Los adolescentes están en una etapa de su vida, donde empieza a cambiar su cuerpo, sus ideales, en otros casos el cambio es negativo al exteriorizar conductas agresivas es decir no controlan sus emociones, generando altos problemas como la agresividad; (Torregrosa, García, Martínez, \& Delgado, 2014), el adecuado manejo de las emociones tiene importancia en el ambiente educativo, al ser una habilidad que se maneja antes las situaciones positivas o negativas con el fin de buscar el bienestar

La influencia de la inteligencia emocional como factor protector frente al desgaste por empatía en terapeutas, ha sido manejada para alcanzar el coeficiente intelectual a través de los diferentes niveles de aprendizaje; (Barbara, 2015), expone que es un elemento 
fundamental que sirve de ayuda al terapeuta para prevenir el desarrollo del desgaste emocional (Barbara, 2015), la atención emocional correspondía a un nivel alto en preocupación empática y al mismo tiempo, hacia los problemas de los demás considerando que pueden existir una relación positiva entre inteligencia emocional, el bienestar psicológico y estabilidad emocional.

Ejecutar ciertas destrezas ayuda en el desarrollo de la inteligencia siendo un factor importante dentro de la psicología que aportara mucho dentro del contexto escolar de los estudiantes como un vector en las habilidades que este va adquiriendo y mejorando su autoestima, conjunto la afectividad como es la felicidad. (Bermúdez, Álvarez, \& Sánchez, 2003).

Según (Sandino, 2003), emplear el enfoque cognitivo conductual sirve de herramienta importante dentro de la psicología por medio de un programa psicoterapéutico grupal para fortalecer la inteligencia emocional, (Cavallo, 1998) es necesario dentro del contexto primario fomentar la afectividad, caso contrario al demostrar una actitud negativa se desfavorece en el desarrollo cognitivo.

(Pérez, 2013), la motivación y la inteligencia emocional son dos importantes aspectos de la educación de los jóvenes que favorecen en las necesidades psicológicas; por lo cual se requiere que en los centros educativos los docentes apliquen estrategias innovadoras para adquirir conocimientos, y mejorar el desarrollo intelectual.

Mediante actividades recreativas se podrá motivar a los escolares para que desarrollen la inteligencia emocional a través de la experimentación de emociones positivas según estudios realizados por (Bermúdez, Álvarez, \& Sánchez, 2003), se ha comprobado que la afectividad favorece en el aprendizaje, la inteligencia, y el rendimiento.

Los factores de la Inteligencia emocional son necesarios como el clima motivacional, con algunas necesidades psicológicas y con las diferentes formas de motivación, los sujetos procesan la información emocional y las ideas de autolesión, de modo que el papel de la inteligencia emocional parece ser un factor importante para proteger a los individuos.

Es importante identificar y comparar las características de la inteligencia emocional en los sujetos con ideas suicidas tomando en cuenta las dificultades en habilidades emocionales necesarias para atender, comprender y regular emociones, contrario a lo que ocurre con quienes no presentan ideas de autolesión; (Shapiro, 1997) considera que el reconocer las emociones negativas desde el aspecto cognitivo y afectivo, permite conocer las debilidades. 
Para prevenir dificultades en el ambiente educativo como: pérdidas de año, baja autoestima, carente integración, desinterés en el aprendizaje, limitaciones en la expresión, en el desarrollo cognitivo, se requiere el establecimiento y determinación de actividades que incrementen la confianza, (García \& García, 2012) caso contrario, al ser manejada de manera incorrecta se convertirá en problemas de salud tales como ansiedad, trastornos del estado de ánimo, problemas psicofisiológicos, que según (López, 2005), dan lugar a alteraciones del sistema inmune, cambios en los hábitos de conductas saludables o mayor prevalencia de conductas adictivas.

La Inteligencia Emocional proporciona las posibilidades de alcanzar procesos que pueden ir de lo simple a lo complejo, en la medida en que interaccionan entre sí cada una de las capacidades que la componen, manteniendo un sentido claro y sincero de las fortalezas y debilidades propias.

Según (López, 2005), el conocimiento de las emociones y el bienestar son aspectos claves para el desarrollo del ser humano, al aportar en la autorregulación, automotivación, empatía, tiempo de métodos asertivos, eficaz el método y juzgarse a sí mismo; (Isaza \& Calle, 2016) las actividades efectuadas en el ambiente educativo y fuera de él, facilita el reconocimiento de habilidades, limitaciones y la sensibilidad hacia el aprendizaje personal.

El reconocimiento de la inteligencia emocional por competencias posibilita al educando interactuar en comunidad con otros a través de las habilidades y destrezas, además, en las relaciones académicas profesor - estudiante son necesarias para que los estudiantes expresen los que piensan prevaleciendo la conciencia emocional, la autoevaluación precisa y la confianza en sí mismos.

\section{Metodología.}

El trabajo tomo como fundamento el enfoque cualitativo y cuantitativo al ser cualitativo contribuyo para mejorar el marco teórico lo que facilito en la operacionalización de las variables, para el análisis e interpretación y posteriormente beneficio en la verificación y comprobación de la hipótesis dando lugar a la determinación de conclusiones y recomendaciones; integra como variable independiente las inteligencia emocional y como variable dependiente el aprendizaje cognitivo.

La indagación toma como modalidades el estudio de campo y el estudio bibliográfico documental que aportaron en el desarrollo cognitivo, al incrementar el conocimiento sobre el problema estudiado, dando lugar al perfeccionamiento de experiencias esenciales para alcanzar la atención, concentración, afectividad e integración del educando hacía en el proceso enseñanza aprendizaje. 
Los tipos de investigación fueron el exploratorio porque aporto en la descripción sistemática para conocer el problema y formular la hipótesis; mientras que el tipo descriptivo a través de tablas y gráficos a portaron en el análisis estadístico para establecer criterios relevantes que fueron fundamentados a nivel explicativo para sintetizar, analizar sobre la importancia de la inteligencia emocional en el aprendizaje cognitivo, además se fortaleció la asociación de las variables para promover una educación de calidad y calidez.

La población estuvo conformada por cuarenta y ocho personas objeto de estudio de los cuales cuarenta y tres fueron estudiantes con un equivalente del ochenta y ocho por ciento y cinco docentes formaron el cinco por ciento es decir se usaron un porcentaje del cien por ciento.

Tabla No. 1. Población investigada.

\begin{tabular}{lcc}
\hline \multicolumn{1}{c}{ Objeto de Estudio } & Frecuencia & Porcentaje \\
\hline Estudiantes & 43 & $88 \%$ \\
Docentes & 5 & $12 \%$ \\
\multicolumn{1}{c}{ Total } & 48 & $100 \%$ \\
\hline
\end{tabular}

Elaborado por: Grupo de investigadores.

Fuente: Grupo de Investigadores.

Después de efectuada la operacionalización de las variables tanto independiente como dependiente se determina las técnicas e instrumentos que favorecerán en la recolección de información enfocada en el mejoramiento de la autoestima, desarrollo emocional, las habilidades emocionales, el pensamiento creativo para alcanzar el desarrollo cognitivo, la comprensión el entendimiento y las relaciones interpersonales; que integran el autocontrol la confianza en sí mismo y la auto regulación, fortaleciendo el control de emociones y las conductas inapropiadas.

Posteriormente se formalizó el análisis e interpretación de datos a través de un cuestionario estructurado que promovió la adquisición de conocimientos que dieron realce a la comprobación de la hipótesis sustentada en el modelo lógico, matemático, estadístico; además se empleó o se usó las fórmulas de los grados de libertad y del chi cuadrado; deduciéndose que las inteligencias emocionales si influyen en el aprendizaje cognitivo, en el desarrollo de capacidades, el manejo de enfrentamientos de manera positiva, el aprendizaje de conocimientos emocionales, la dirección del trabajo en equipo y un buen liderazgo.

Tabla N. 2. Frecuencias Observadas (Recolección de datos).

PREGUNTAS

$\begin{array}{llll}\text { SIEMPRE } & \text { A VECES NUNCA } & \text { SUBTOTAL }\end{array}$


Pregunta $N^{0}$. 2: ¿Usted mantiene el autocontrol de las emociones, demostrando confiabilidad a sumiendo las responsabilidades de su desempeño académico?

10

Pregunta No. 7: ¿Usted mediante la comprensión y reflexión enfrenta situaciones conflictivas que se presentan en el diario vivir, exteriorizando una actitud crítica?

Pregunta No. 8: ¿Usted aplica conocimientos de diferentes disciplinas para la toma de decisiones asertivas y socialmente responsables, a partir de un proceso de análisis?

Pregunta $N^{\mathbf{0}}$. 10: ¿Usted ha desarrollado la percepción, la memoria, el razonamiento y la reflexión, disminuyendo las dificultades en el aprendizaje?

SUBTOTALES
16

22

17

61
33

23

20

25

105
0

4

43

43

Fuente: Encuesta.

Elaborado por: grupo de investigadores

Un mayor porcentaje de encuestados a veces muestran interés asía las responsabilidades, lo que limita en el manejo de las destrezas; mientras un menor porcentaje, ejecuta el control de las emociones, con llevando a la responsabilidad actividades escolares; no comparte información, lo que perjudica en el desarrollo de habilidades cognitivas; mientras un menor porcentaje, ha fortalecido la inteligencia emocional, con llevando a la responsabilidad en el cumplimiento de actividades escolares y extraescolares.

Los educandos fortalecen el liderazgo fomentan los lazos de amistad; algunos estudiantes muestran actitudes empáticas dentro del aula, que influye en la toma de decisiones, lo que limita en el manejo de las destrezas; mientras una parte manifiesta que siempre ejerce una influencia en su entorno, mostrando interés por ayudar, abre oportunidades para comprender el medio social, para aprender sobre el mundo, maneja las capacidades de atención y memoria, emplea los mecanismos perceptivos, desarrolla el aprendizaje cognitivo, alcanza el trabajo en cooperación estimula la sociabilización y logra el éxito personal como emocional.

\section{Resultados.}

Después de realizada la indagación a los estudiantes, se observó el 58\% a veces manejan el autocontrol de las emociones, demuestran la confiabilidad y asumen responsabilidades en su desempeño académico; el $40 \%$ siempre demuestra autonomía en el cumplimiento de tareas; mientras el $60 \%$ a veces busca el entendimiento, comparte información.

Se deduce que el $58 \%$ fortalecen el trabajo en equipo dentro del salón de clases; el $42 \%$ a veces adopta una actitud empática y social potencia sus posibilidades de desarrollo 
personal; el $60 \%$ no comunica sus pensamientos e ideas, no aporta en la solución de problemas, lo que condiciona el trabajo en equipo y la exteriorización del conocimiento.

El 42\% siempre desarrolla la capacidad de liderazgo, propicia un adecuado ambiente de trabajo, orienta y dirige a los demás para motivarlos, el 58\% no coordinan y organizan los grupos de trabajo; lo que desfavorece en el refuerzo de relaciones interpersonales, disminuye el alcance de metas compartidas; además el 65\% no cumple con las tareas en el tiempo estipulado, lo que limita el aprendizaje cognitivo, el pensamiento crítico, la exteriorización de ideas con significancia.

Se determina que el 56\% no ha desarrollado conocimientos relacionados a diferentes disciplinas, es carente la toma de decisiones asertivas y la articulación con el análisis y la síntesis; factores concionantes para el autoestima, el aprendizaje, la convivencia y la interrelaciones entre los integrantes de la comunidad educativa.

El 58\% es consciente de expresar sus propias ideas, aporta con nuevas ideas; emiten juicios críticos, toma decisiones con perspectivas de acuerdo a la temática; mientras el $46 \%$ no comprende y reflexiona ante conflictos en el contexto; es decir no enfrenta situaciones complicadas, evade su responsabilidad, demostrando una carente confianza en sí mismo, lo que repercute en el pensamiento creativo; y el 58\% no ha desarrollado la percepción, los conocimientos y habilidades, desfavoreciendo en la memoria, entendimiento y reflexión presentado dificultades en el aprendizaje cognitivo.

\section{Discusión.}

Para (Ibáñez \& Mudarra, 2014), la gestión educativa se basa en los procesos pedagógicos para el mejoramiento de cada una de sus áreas, a través del liderazgo lo cual permitirá a los estudiantes desarrollar sus aptitudes tomando como herramienta fundamental la inteligencia emocional; (Delgado, 2013) el carente fortalecimiento de la inteligencia emocional perjudica en la innovación, el desarrollo intelectual y el aprendizaje; (Cassullo \& García, 2015), en el ambiente educativo la atención aumenta la claridad de los sentimientos y emociones, que permite vivir de forma contemplativa los sentimientos, acciones y pensamientos.

(Cassullo \& García, 2015), considera que la familia es un eje principal donde se centra las atenciones y permite favorecer un clima agradable para desarrollas las emociones en el pensamiento, comprender y razonar con emoción y regular las emociones, mientras (Ramos, Herrera, \& Ramírez, 2015), expone que la familia busca el bienestar de los hijos a través de la socialización de las competencias emocionales. 
El carente desarrollo de habilidades cognitivas favorece en la solución de problemas, toma de decisiones, pensamiento crítico, pensamiento creativo, por lo cual (Yánez, 2016), expone que las etapas del proceso de aprendizaje se realiza de manera gradual e interconectada con la motivación, interés, atención, adquisición, comprensión, asimilación, aplicación, transferencia y evaluación, por media de actividades cotidianas de docencia en diferentes niveles educativos.

Para (Ramón, 2017), el desarrollo cognitivo está asociado con el aprendizaje significativo, siendo el proceso por el que una persona va adquiriendo conocimientos sobre lo que le rodea y desarrolla su inteligencia y capacidades, en apoyo (Yánez, 2016) expresa que el aprendizaje significativo es alcanzado a través de la confianza y el apoyo familiar; (Molano, 2012) agrega que el educando a través del desarrollo de la inteligencia construye formas para pensar, sentir y hacer; (Ramón, 2017) incrementa que los conocimientos sólidos, son alcanzados a través de la práctica y la experiencia.

En referencia a los criterios expuestos, la inteligencia emocional es un potencial que promueve las relaciones interpersonales y el bienestar psicológico, además la integración de la familia para el aprendizaje cognitivo favorece en la atención emocional a través de la cohesión, organización, expresividad e implicación en actividades intelectual-culturales, lo que incentiva en la seguridad de sí mismo, la confianza y el progreso de desarrollo de la relación mente-emociones-cuerpo donde algunos autores consideran que aún la relación entre bienestar general y consciencia ha recibido poca atención empírica .

El investigador (Molano, 2012), en el trabajo del método afectivo cognitivo para el aprendizaje, señala que el docente a través de la selección del material mejora las habilidades para que los educándose incrementen sus fortalezas; mientras (López O. , 2014), expone que el trabajo en unión con los padres favorece el desarrollo de competencias para la autonomía basadas en modelos de autorregulación, en la que la articulación de factores motivacionales y el uso de estrategias de aprendizaje

Para (Delgado, 2013) en el desarrollo del aprendizaje se encuentra la motivación, rendimiento, cuidado, adquisición, conocimiento, asimilación, concentración, transmisión y estimación que ayudan en el manejo apropiado del aprendizaje; (Molano, 2012) agrega que el aprendizaje cognitivo es una manera innovadora para enseñar al requerirse de habilidades cognitivas; (López O. , 2014) deduce que la solución de problemas, toma de decisiones, pensamiento crítico, es alcanzado mediante la integración, interrelación, y afectividad que el educando reciba en sus diferentes ambientes.

Las estrategias de aprendizaje como la interacción social son subyacentes a la disposición de aprendizaje por parte del estudiante es decir el incremento del conocimiento adaptativo 
del aprendiz al medio físico, social, histórico y cultural, producto del aprendizaje significativo de la conceptualización científica en un proceso de representación mental; siendo el aprendizaje cognitivo obtenido mediante la autoeficacia es un factor motivacional que impulsa a los a persistir frente a las dificultades, aumenta el esfuerzo y ayuda en la planificación de actividades; es así como la enseñanza y el aprendizaje aportan para una educación de calidad, eficiencia y productiva.

\section{Conclusión.}

- En la Unidad Educativa P.C.E.I. San Miguel, se ha observado en los estudiantes un escaso control de emociones limitando a que puede ejecutar sus habilidades y destrezas cognitivas dentro del cumplimiento de actividades; también los estudiantes demuestran un carente liderazgo dentro del aula, generando un clima inadecuado para el aprendizaje cognitivo.

- Dentro de la investigación es evidente demostrar la escasa influencia en la toma de decisiones, lo que está perjudicando el dominio del lenguaje, con llevando a que no puedan desarrollar de la mejora manera su aprendizaje cognitivo; además los educandos no adquieren con facilidad sus conocimientos a través de experiencias, lo que desmotiva en el aprendizaje y pensamiento crítico en la integración e inteligencia emocional.

- Se determina que las personas encuestadas en un porcentaje del $56 \%$ exteriorizan que la inteligencia emocional al no ser fortalecida mediante actividades, tareas, repercute en el control de emociones que afecta en el aprendizaje cognitivo; de igual manera limita la expresión de ideas propias, genera barreras para el alcance del aprendizaje cognitivo.

- Después de aplicada la encuesta a los educandos y la entrevista a los docentes se deduce que $42 \%$ consideran que es importante la inteligencia emocional para alcanzar el aprendizaje cognitivo; lamentablemente el 58\% no han desarrollado las capacidades en: atención, confianza, seguridad, y autoestima para mejorar el desempeño académico; además, es carente el control de las emociones lo que incrementa la inseguridad; el 60\% exterioriza que la escasa aplicación de actividades que fortalezcan la inteligencia emocional han con llevado al incumpliendo de tareas escolares y extraescolares limitando el alcance de una educación con calidad

- Se deduce que entre las causas influyentes para el aprendizaje cognitivo en un 58\% establecen el inadecuado trabajo en equipo la escasa actitud empática, el carente 
manejo de conflictos; mientras un porcentaje del $60 \%$ deduce que es insuficiente la comprensión para realizar los trabajos en el aula lo que ha incrementado la desorientación para la resolución de dificultades familiares, incrementando la deficiente autoconfianza, autovaloración y conciencia emocional; desmotivación en el alcance de objetivos, escaso compromiso, iniciativa y optimismo.

- Se determina en un $56 \%$ que la inexistencia de un documento que vincule las variables de estudio en un $56 \%$ ha perjudicado en las relaciones personales, la interacción y en el lenguaje, el 65\% exterioriza la carencia en el proceso de análisis, la emisión de juicios críticos y la toma de decisiones; el 58\% expone la necesidad de elaborar un escrito que aporte en el conocimiento de la inteligencia emocional y en el aprendizaje cognitivo para mejorar el proceso de enseñanza y aprendizaje

\section{REFERENCIAS BIBLIOGRÁFICAS}

1. Alviárez, L., \& Pérez, M. (2009). Inteligencia emocional en las relaciones académicas. Distrito Federal, México: Laurus.

2. Andrade Guambaña, S. M. (2014). El desarrollo de la Inteligencia Emocional en el proceso de enseñanza - aprendizaje de los niños de séptimo año de Educación Básica de la Unidad Educativa Santana de la ciudad de Cuenca en el año lectivo 2013-2014. Cuenca: Universida Politécnica Salesiana.

3. Asamblea Constituyente. (2008). Constitución Política de la República del Ecuador. Quito, Ecuador: Ruiz. Obtenido de Marco legal: http://www.asambleanacional.gob.ec/sites/default/files/documents/old/constitucion_ de_bolsillo.pdf

4. Barbara, A. (2015). Inteligencia emocional y desgaste por empatía en terapeuta. Buenos Aires, Argentina: Clínica Psicológica.

5. Barragán, M. (2015). Pedagogía, aprendizaje y competencias emocionales. La Habana, Cuba: Pueblo y Educación.

6. Bello, Z., Rionda, D., \& Rodríguez, M. (2010). Inteligencia y pedagogía para el edsarrollo emocional de los educandos. Barcelona, España: Paidós.

7. Bermúdez, M. P., Álvarez, T., \& Sánchez, A. (2003). Análisis de la relación entre inteligencia emocional, estabilidad emocional y bienestar psicológico. Zaragoza, España: Universitas Psychologica.

8. Bofill, P. \&. (2007). Las fases del aprendizaje: Un esquema para el análisis y diseño. En Bofill, P., \& Miró, J. Las fases del aprendizaje: Un esquema para el análisis y diseño de actividades de enseñanza/aprendizaje. (pág. 2). Barcelona.

9. Boterf, L. (2014). Educación emocional y competencias básicas para la vida. Revista de Investigación Educativa (RIE), 1-43. 
10. Boyatzis Richard. Mckee Annie. (2016). El lider resonante crea mas. España.

11. Brackett, M., Rivers, S., Shiffman, S., Lerner, N., \& Salovey, P. (2005). ¿Cuál es la mejor manera de medir la inteligencia emocional? Nueva York, Estados Unidos: Nova Science.

12. Bravo, F. (20 de 01 de 2013). Aprendizaje cognitivo. Obtenido de Slide Share: https://es.slideshare.net/afelipebravom/aprendizaje-cognitivo-16089269

13. Caballo, V. (2002). Manual de evaluación y entrenamiento de las habilidades sociales. Barcelona, España: S. XXI.

14. Cándido, J., TOrregrosa, M., García, J., Martínez, M., \& Delgado, E. (2014). Conducta agresiva e inteligencia emocional en la adolescencia. Murcia, España: Jornada de Educación Y psicología.

15. Carrillo, D. (2015). Desarrollo de competencias emocionales en el ambiente familiar, escolar y social. Palma de Mallorca, España: Torremozas.

16. Cassullo, G. L., \& García, L. (2015). Estudio de las competencias socio emocionales y. (España, Ed.) Madrid: TEA.

17. Castilla Pérez, M. F. (2014). La teoría del desarrollo cognitivo de Piaget aplicada en la clase de Primaria. Uva doc.

18. Cataldo, C. (2014). Desarrollo infantil: el cognitismo, la inteligencia y la memoria. La Habana, Cuba: Pueblo Nuevo.

19. Cavallo, V. (1998). Modificación de conducta a traves de la confianza en sí mismo. Madrid, España: Siglo XXI.

20. Chico Villagrán, M. A. (2016). La inteligencia emocional en el rendimiento escolar de los estudiantes de 4to, 5to, 6to año de educación general básica, de la "Unidad Educativa Ambato" de la ciudad de Ambato provincia de Tungurahua. Ambato, Ecuador: Universidad Técnica de Ambato.

21. Código de la Niñez y Adolescencia. (2016). Título III. Derechos, garantías y deberes; Capítulo III. Derechos relacionados con el desarrollo. Quito, Ecuador: Lexis S.A.

22. Corrales, G. (11 de septiembre de 2013). Periódico Cotopaxi: Noticias las estadísticas de suicidios . Actividades de Salud Mental de la Dirección Provincial de Salud, pág. 1.

23. Damiel, G. (2010). Inteligencia Emocional. Española.

24. Delgado, M. (2013). Aplicación didáctica de las inteligencias múltiples. Buenos Aires, Argentina: Kapelusz.

25. Echeverría, J. (2014). Proceso de enseñanza aprendizaje. Madrid, España: Narcea.

26. Extremera Pacheco, N., \& Fernández-Berrocal, P. (2004). El papel de la in teligencia emcional en los escolares. Madrid, España: Graó.

27. Fernández Macías, N. M. (2010). La multimedia en el aula y su influencia en el aprendizaje cognitivo del idioma inglés de los alumnos de décimo año de educación 
básica de la Unidad Educativa experimental "Pedro Fermín Cevallos" del cantón Ambato, en el periodo junio octubre/2010. Ambato: Universidad Tecnica Ambato.

28. Fernández Macías, N. M. (2010). La multimedia en el aula y su influencia en el aprendizaje cognitivo del idioma inglés de los alumnos de décimo año de educación básica de la Unidad Educativa experimental "Pedro Fermín Cevallos" del cantón Ambato, en el periodo junio octubre/2010. Ambato, Ecuador: Universidad Técnica de Ambato.

29. Fernández, P., \& Ruiz, D. (2008). Inteligencia emocional en el rendimiento academico y el sistema educativo. Barcelona, España: Paidós.

30. Friedlander, E. (1996). Educar con Inteligencia Emocional. Barcelona, España: Plaza y Jané.

31. Friedlander, E. (1996). Educar con Inteligencia Emocional. Barcelona, España: Plaza y Jané.

32. Gallego, A. (2011). El contructo de la Inteligencia Emocional importante en sus repercusiones pedagógicas. España.

33. García, J., \& García, Á. (2012). La Inteligencia Emocional como estrategia de prevención de las adicciones. Concepción, Chile: Latinoamericana de Psicología.

34. García, J., \& Nieto, A. (2009). La Dimensión Comunicativa de las Inteligencias Múltiples. Cuadernos de Información y Comunicación. Madrid, España: Paidós.

35. Gardner, H. (1996). Las inteligencias interpersonal e intrapersonal.

36. Gardner, H. (2005). Inteligencias Múltiples, la Teoría e la Práctica. Bogotá: Surcos.

37. Gesell, A. (1985). Inteligencia emocional en el aprendizaje infantil y el desarrollo e competencias. Madrid, España: Paidós Ibérica.

38. Gonzales, L. y. (2013). Inteligencia.

39. Ibáñez, I., \& Mudarra, M. (2014). Atención temprana. Diagnóstico e intervención psicopedagógica. Madris, España: UNED.

40. Isaza, G., \& Calle, S. (2016). Acercamiento a la comprensión del pñerfil de la inteligencia emocional. Murcia, España: Revista Latinoamericana de Ciencias Sociales.

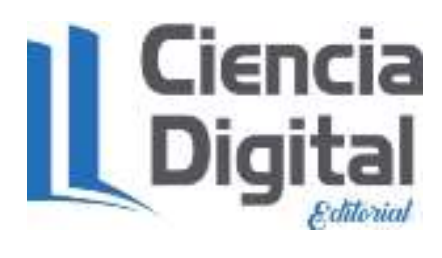




\section{Para citar el artículo indexado.}

Andrade N., Andrade J., Andrade N, Peñafiel V. \& Andrade. A. (2018). Perspectivas de la inteligencia emocional y desarrollo del aprendizaje cognitivo. Revista electrónica Ciencia Digital 2(1), 345-359. Recuperado desde: http://www.cienciadigital.org/revistascienciadigital2/index.php/CienciaDigital/article/view/ $24 / 24$

\section{【 Ciencia}

El artículo que se publica es de exclusiva responsabilidad de los autores y no necesariamente reflejan el pensamiento de la Revista Ciencia Digital.

El articulo queda en propiedad de la revista y, por tanto, su publicación parcial y/o total en otro medio tiene que ser autorizado por el director de la Revista Ciencia Digital.
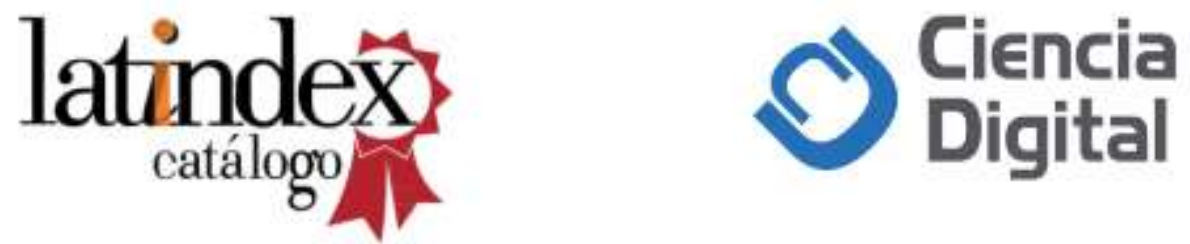